\title{
Programas de intervenção na Síndrome de Burnout: uma revisão integrativa de literatura
}

\author{
Burnout Syndrome intervention programs: an integrative literature review \\ Programas de intervención en el Síndrome de Burnout: una revisión bibliográfica \\ integradora
}

Izabela Souza da Silva ${ }^{1 *}$, Lúcyo Wagner Torres de Carvalho', Geraldo Magella Teixeira1, Maria Goretti Fernandes ${ }^{2}$, Almira Alves dos Santos ${ }^{1}$, Silvia Pessoa de Freitas Pedrosa de Oliveira ${ }^{1}$, Geraedson Aristides da Silva1.

\section{RESUMO}

Objetivo: Identificar e analisar as produções científicas existentes sobre os programas de intervenção na Síndrome de Burnout (SB). Métodos: Tratou-se de uma revisão integrativa da literatura, em que a busca por artigos foi realizada nos meses de agosto e setembro de 2019, nas bases de dados eletrônicas: Scientific Electronic Library Online (SCIELO), Biblioteca Virtual em Saúde (BVS) e National Library of Medicine (PubMed). Resultados: Foram selecionados 22 artigos para compor esta revisão. 59,1\% dos estudos analisados foram publicados nos últimos 02 anos; $43,5 \%$ realizados na América do Norte e 68,2\% efetuadas com profissionais de saúde. Praticamente em todos os estudos analisados foram empregadas estratégias de prevenção/enfretamento da SB com foco no trabalhador e $81 \%$ deles demonstraram algum benefício na prevenção/terapêutica da SB. Considerações finais: Foi identificado que estudos sobre SB têm crescido ao longo dos anos, sobretudo na comunidade cientifica internacional e, apesar de ser um tema ainda pouco explorado no Brasil, vale destacar que houve uma equivalência no tocante aos instrumentos utilizados para detecção e prevenção da SB nos estudos que compuseram a presente pesquisa. Ressalta-se a necessidade de novos estudos, pois ainda há uma carência dessas pesquisas tanto em âmbito nacional quanto internacional.

Palavras-chave: Burnout, Prevenção, Programa.

\section{ABSTRACT}

Objective: To identify and analyze the existing scientific productions about the intervention programs in Burnout Syndrome (SB). Methods: This was an integrative literature review, in which the search for articles was carried out in the months of August and September 2019, in the electronic databases: Scientific Electronic Library Online (SCIELO), Virtual Health Library (VHL) and National Library of Medicine (PubMed). Results: 22 articles selected to compose this review. $59.1 \%$ of the analyzed studies were published in the last 02 years; $43.5 \%$ carried out in North America and $68.2 \%$ carried out with health professionals. In virtually all of the studies analyzed, prevention / coping strategies for BS focused on the worker were used, $81 \%$ of them demonstrated some benefit in preventing / treating BS. Final considerations: It was identified that studies on BS have grown over the years, especially in the international scientific community and, despite being a topic still little explored in Brazil, it is worth mentioning that there was an equivalence regarding the instruments used for detection and prevention SB in the studies that comprised the present research. The need for further studies is emphasized, as there is still a lack of such research both nationally and internationally.

Key words: Burnout, Prevention, Program.

\section{RESUMEN}

Objetivo: Identificar y analizar las producciones científicas existentes sobre los programas de intervención en el Síndrome de Burnout (SB). Métodos: Se realizó una revisión bibliográfica integradora, la búsqueda de artículos se realizó en agosto y septiembre de 2019, en las bases de datos electrónicas: Biblioteca Electrónica Científica en línea (SCIELO), Biblioteca Virtual en Salud (BVS) y Biblioteca Nacional de Medicina (PubMed). Resultados: Se seleccionaron 22 artículos para componer esta revisión. $59,1 \%$ de los estudios analizados se publicaron en los últimos 02 años; $43.5 \%$ realizado en Norteamérica y $68.2 \%$ realizado con profesionales de la salud. En prácticamente todos, se utilizaron estrategias de prevención/afrontamiento para BS centradas en el trabajador, $81 \%$ de ellas demostraron algún beneficio en la prevención/tratamiento de BS. Consideraciones finales: Se identificó que los estudios sobre BS han crecido a lo largo de los años, especialmente en la comunidad científica internacional, a pesar de ser un tema aún poco explorado en Brasil, vale mencionar que hubo una equivalencia con respecto a los instrumentos utilizados para la detección y prevención. SB en los estudios que comprendieron la presente investigación. Se enfatiza la necesidad de realizar más estudios, ya que todavía existe falta de investigación a nivel nacional e internacional.

Palabras clave: Agotamiento psicológico, Prevención, Programa.

1Universidade Estadual de Ciências da Saúde de Alagoas (UNCISA), Maceió - AL. *E-mail: izabellapsi100@gmail.com

2Universidade Federal de Sergipe (UFS), Aracaju - SE.

SUBMETIDO EM: 6/2020

ACEITO EM: 7/2020

PUBLICADO EM: 10/2020 


\section{INTRODUÇÃO}

O mundo do trabalho contemporâneo - dados os desafios do desenvolvimento industrial, da globalização, do avanço tecnológico e da comunicação virtual - impõe a classe trabalhadora condições que, muitas vezes, excedem os limites de suas habilidades e capacidades. O resultado é o estresse no ambiente laboral, que pode acarretar disfunções físicas, psicológicas e até sociais, prejudicando a saúde do trabalhador, interferindo na sua capacidade produtiva e, até mesmo, refletindo de forma destrutiva na sua vida pessoal e na sua interação para com as pessoas (OPAS, 2016).

Segundo a International Labour Organization (2016), essas disfunções podem variar de acordo com o indivíduo. Todavia, níveis elevados de estresse podem contribuir para o desenvolvimento de deficiências físicas, como doenças cardiovasculares e distúrbios musculoesqueléticos; desencadear comportamentos emergentes de enfrentamento, como o abuso de álcool e outras drogas, além de problemas mentais e distúrbios comportamentais como exaustão, ansiedade, depressão e burnout.

Dados da Organização Mundial da Saúde (OMS) (2019a), declaram que a SB, também conhecida como síndrome do esgotamento profissional, está descrita na Classificação Estatística Internacional de Doenças(CID-11) como um fenômeno ocupacional resultante de um estresse laboral crônico que não foi gerenciado com sucesso, não devendo ser empregada para descrever experiências em outras áreas da vida. A SB caracteriza-se por três dimensões: sentimentos de exaustão ou esgotamento de energia; aumento do distanciamento mental do próprio trabalho, ou sentimentos de negativismo ou cinismo relacionados ao próprio trabalho; e redução da eficácia profissional (MASLACH C, et al., 1996).

No Brasil, o decreto no 3.048 (1999), que regulamenta a Previdência Social, considera a síndrome do esgotamento profissional como doença do trabalho. Ressalta-se que, como os casos de SB não enquadram no rol de doenças que exigem notificação compulsória, o Ministério da Saúde não consegue estimar sua prevalência. Todavia, estudo realizado pelo International Stress Management Association (ISMA-BR) (2017), estimou que $30 \%$ dos trabalhadores brasileiros sofrem com a síndrome. Tal proporção é análoga à do Reino Unido, país no qual 1 a cada 3 habitantes (o equivalente a 20 milhões de pessoas) enfrenta o problema (ISMABR, 2017).

Segundo a OMS (2019b), as empresas podem realizar intervenções como parte de uma estratégia integrada para promoção da saúde e bem-estar que incluam aspectos preventivos, de identificação precoce, apoio e reabilitação. Essas intervenções podem ser direcionadas aos trabalhadores (aplicadas individualmente ou em grupo, constituem-se em medidas comportamentais que visam melhorar, dentre outros aspectos, a capacidade profissional e habilidades pessoais de enfrentamento), à organização (promovendo alterações nos sistemas de trabalho, por meio da reestruturação, avaliação e supervisão das tarefas, visando diminuir a sobrecarga de trabalho e aumentar o controle ou o nível participativo na tomada de decisão) ou mistos (quando abrangem ambos aspectos) (AWA WL, et al., 2010).

Assim sendo, no intuito de compreender melhor e contribuir com o tema fomentando discussões relacionadas com a saúde dos trabalhadores e a implicação desta para o ambiente ocupacional, o propósito do presente estudo é identificar e analisar as produções científicas existentes sobre os programas de intervenção na Síndrome de Burnout.

\section{MÉTODOS}

A presente pesquisa trata-se de uma revisão integrativa de literatura, que é um método da Prática Baseada em Evidências (PBE). Para o desenvolvimento dessa revisão integrativa, foram percorridas 06 etapas distintas, a saber: (1) identificação do tema e formulação da questão norteadora; (2) determinação dos critérios de inclusão e exclusão dos estudos para a realização da pesquisa; (3) categorização dos estudos, definindo as informações a serem extraídas dos estudos selecionados; (4) avaliação dos estudos incluídos na revisão; (5) interpretação dos resultados e (6) apresentação da revisão através da síntese do conhecimento evidenciado nos artigos analisados.

Assim sendo, delimitou-se para o presente estudo o seguinte tema: "Programas de intervenção empregados na prevenção/terapêutica da Síndrome de Burnout", sendo formulada a seguinte pergunta condutora: "Quais os instrumentos de aferição do estresse ocupacional crônico, mais conhecido, como 
Síndrome de Burnout, os programas de intervenção empregados na sua prevenção/terapêutica e os benefícios dessas intervenções para saúde do trabalhador e/ou ambiente laboral?"

Realizou-se a busca por publicações nos meses de agosto e setembro de 2019. O levantamento bibliográfico dos estudos baseou-se no material indexado nas bases de dados eletrônicas: SCIELO, BVS e PubMed. Para o levantamento dos artigos foram utilizados os seguintes termos/palavras-chaves: "Burnout", "Prevenção" e "Programa", e suas respectivas traduções nos idiomas inglês e espanhol, que foram combinados em cada base de dados (utilizou-se "and" como operador booleano entre os termos).

Foram incluídos na pesquisa artigos, monografias, dissertações e teses indexados nas referidas bases eletrônicas de dados que se encontravam disponíveis de modo completo e gratuito nos meios eletrônicos acima descritos nos idiomas português, espanhol e inglês que tratavam do tema em análise. Foi estabelecido como limite de tempo para busca de publicações os últimos 5 anos (2015-2019).

Excluíram-se da pesquisa, livros e capítulos de livros, pela possível dificuldade de acesso a essas obras; revisões de literatura; e estudos que tratavam de outras formas de estresse ocupacional; que não apresentavam os resultados do programa de intervenção utilizado na prevenção/terapêutica da SB; que foram escritos em outros idiomas e/ou que não atendessem aos pressupostos do presente tema.

Para sistematização da avaliação dos selecionados e extração das informações, elaborou-se um instrumento para a coleta de dados composto pelos seguintes itens: autores, título, banco de dados, ano de publicação, idioma, tipo de estudo, local de origem da pesquisa, objetivos do estudo, público-alvo, instrumento de aferição utilizado na identificação da SB, tipo de intervenção, principais resultados e principais conclusões. Os artigos foram classificados e os resultados encontrados foram posteriormente sintetizados, considerando a similaridade de conteúdo.

As publicações foram categorizadas em 03 categorias: instrumentos de aferição da SB, programas de intervenção utilizados na prevenção/terapêutica da SB e benefícios dos programas de intervenção na SB. A interpretação dos resultados obtidos e a síntese do conhecimento evidenciado nos artigos analisados serão apresentadas a seguir.

\section{RESULTADOS E DISCUSSÃO}

\section{Caracterização das publicações}

Por meio de busca eletrônica foram encontradas 291 publicações (25 artigos na base de dados SciELO, 39 na BVS, e 227 na PUBMED). Após aplicação dos critérios previamente estabelecidos, foram selecionados 22 artigos que abordavam programas de intervenção frente à SB (02 da base de dados SciELO, 02 da BVS, e 18 da PUBMED). A seguir apresentar-se-á um panorama geral das publicações (Quadro 1).

Verificou-se que $59,1 \%(n=13)$ estudos foram publicados nos últimos 02 anos. Pesquisas sobre a Síndrome de Burnout tem despertando interesse ao longo dos anos, principalmente por parte da comunidade científica internacional, haja vista que a manifestação da síndrome (esgotamento físico e emocional em função do trabalho) tem sido cada vez mais comum em diversas categorias profissionais (PASTURA PSVC, et al., 2019; HART D, et al., 2019; CARVALHAIS FR, et al., 2015). Configurando, assim, como um importante problema biopsicossocial nos tempos modernos em razão de suas consequências individuais e coletivas (RUBACK SP, 2019).

Quanto ao local de publicação das pesquisas, 43,5\% $(n=10)$ dos estudos foram realizados na América do Norte (todos nos EUA), 21,7\% ( $n=5)$ na Europa, 17,4\% ( $n=4)$ na América do Sul (todos no Brasil), 8,7\% ( $n=2)$ na Àsia e os $8,7 \%$ restantes na Oceania $(n=1)$ e na África $(n=1)$. Países da América do Norte e na Europa tem uma longa tradição no tocante as pesquisas sobre Burnout (CARLOTTO MS e CÂMARA SG, 2008). No Brasil, o tema ainda permanece pouco explorado, principalmente, quando comparado com a literatura internacional (MELO LP e CARLOTTO MS, 2017).

Com relação a metodologia utilizada, os estudos controlados randomizados e os estudos de viabilidade foram os desenhos de estudos mais empregados (ambos com 18,2\%, $n=4$ ). Os estudos randomizados são considerados o padrão de excelência em pesquisas que pretendem avaliar o efeito de uma intervenção no curso de uma situação clínica estabelecida, pois permitem eliminar diversos vieses, em virtude dos grupos 
intervenção e controle serem alocados de forma aleatória e as características serem distribuídas de um modo semelhante entre os dois grupos (BUEHLER AM, et al., 2009).

Por fim, quanto a população estudada, $68,2 \%(n=15)$ das pesquisas foram efetuadas com profissionais de saúde. A SB é mais frequente em trabalhadores cuja natureza de seu trabalho está diretamente relacionada ao atendimento intenso e frequente de indivíduos que necessitam de algum tipo de assistência, geralmente, ocupações que exigem rápida resolutividade, e níveis consideráveis de empatia, cuidado e atenção com outras pessoas, como é o caso dos profissionais de saúde, da educação e da segurança pública (PEREIRA AMB, 2010).

Verificou-se que $59,1 \%(n=13)$ dos estudos foram publicados nos últimos 02 anos. Pesquisas sobre a SB têm despertando interesse ao longo dos anos, principalmente por parte da comunidade científica internacional, haja vista que a manifestação da síndrome (esgotamento físico e emocional em função do trabalho) tem sido cada vez mais comum em diversas categorias profissionais (PASTURA PSVC, et al., 2019; HART D, et al., 2019). Configurando, assim, um importante problema biopsicossocial nos tempos modernos em razão de suas consequências individuais e coletivas (RUBACK SP, 2019).

Nesta perspectiva, pode-se observar que apesar de constituir uma atividade que engrandece o homem, a depender das condições em que se realizam as atividades laborais, o trabalho pode expor o profissional a uma série de riscos, que a longo prazo refletem de forma negativa na vida destes profissionais resultando na SB com forte impacto na vida pessoal e profissional, gerando importantes perdas tanto para o trabalhador quanto para as instituições, que perdem na produtividade à medida que este fenômeno leva os profissionais ao afastamento laboral por tempo indeterminado (MELO LP e CARLOTTO MS, 2017).

Quanto ao local de publicação das pesquisas, 43,5\% ( $n=10)$ dos estudos foram realizados na América do Norte (todos nos EUA), 21,7\% ( $n=5)$ na Europa, 17,4\% $(n=4)$ na América do Sul (todos no Brasil), 8,7\% ( $n=2)$ na Ásia e os $8,7 \%$ restantes na Oceania $(n=1)$ e na África $(n=1)$. Países da América do Norte e da Europa têm uma longa tradição no tocante as pesquisas sobre Burnout. No Brasil, o tema ainda permanece pouco explorado, principalmente, quando comparado com a literatura internacional (MELO LP e CARLOTTO MS, 2017).

Com relação a metodologia utilizada, os estudos controlados randomizados e os estudos de viabilidade foram os desenhos de estudos mais empregados (ambos com 18,2\%, $n=4$ ). Os estudos randomizados são considerados o padrão de excelência em pesquisas que pretendem avaliar o efeito de uma intervenção no curso de uma situação clínica estabelecida, pois permitem eliminar diversos vieses, em virtude dos grupos intervenção e controle serem alocados de forma aleatória e as características serem distribuídas de um modo semelhante entre os dois grupos (BUEHLER AM, et al., 2009).

Quanto a população estudada, $68,2 \%$ ( $n=15)$ das pesquisas foram efetuadas com profissionais de saúde. A SB é mais frequente em trabalhadores cuja natureza de seu trabalho está diretamente relacionada ao atendimento intenso e frequente de indivíduos que necessitam de algum tipo de assistência, geralmente, ocupações que exigem rápida resolutividade, e níveis consideráveis de empatia, cuidado e atenção com outras pessoas, como é o caso dos profissionais de saúde, da educação e da segurança pública (PEREIRA AMB, 2010).

Por fim, foi observado um maior percentual feminino entre os participantes das pesquisas que compuseram o presente estudo. Tal situação pode relacionar-se ao fato do gênero estar inserido cada vez mais no mercado de trabalho; além disso, as obrigações com os afazeres domésticos e os cuidados com os filhos, ainda permanecem sob a responsabilidade de grande parte das mulheres e, o somatório de tais atividades, refletem em uma dupla jornada com consequentes fontes de estresse (CLEMENTE LA, et al., 2015; MEHRABI T, et al., 2015).

\section{Caracterização dos resultados dos estudos}

No tocante aos resultados desses estudos, os mesmos foram agrupados e discutidos em 03 categorias temáticas: instrumentos de aferição da SB, programas de intervenção utilizados na prevenção/terapêutica da SB e benefícios dos programas de intervenção na SB. O detalhamento desses dados segue apresentado no quadro abaixo (Quadro 2). 
Quadro 1 - Descrição dos artigos incluídos na revisão integrativa. Maceió - AL, 2019.

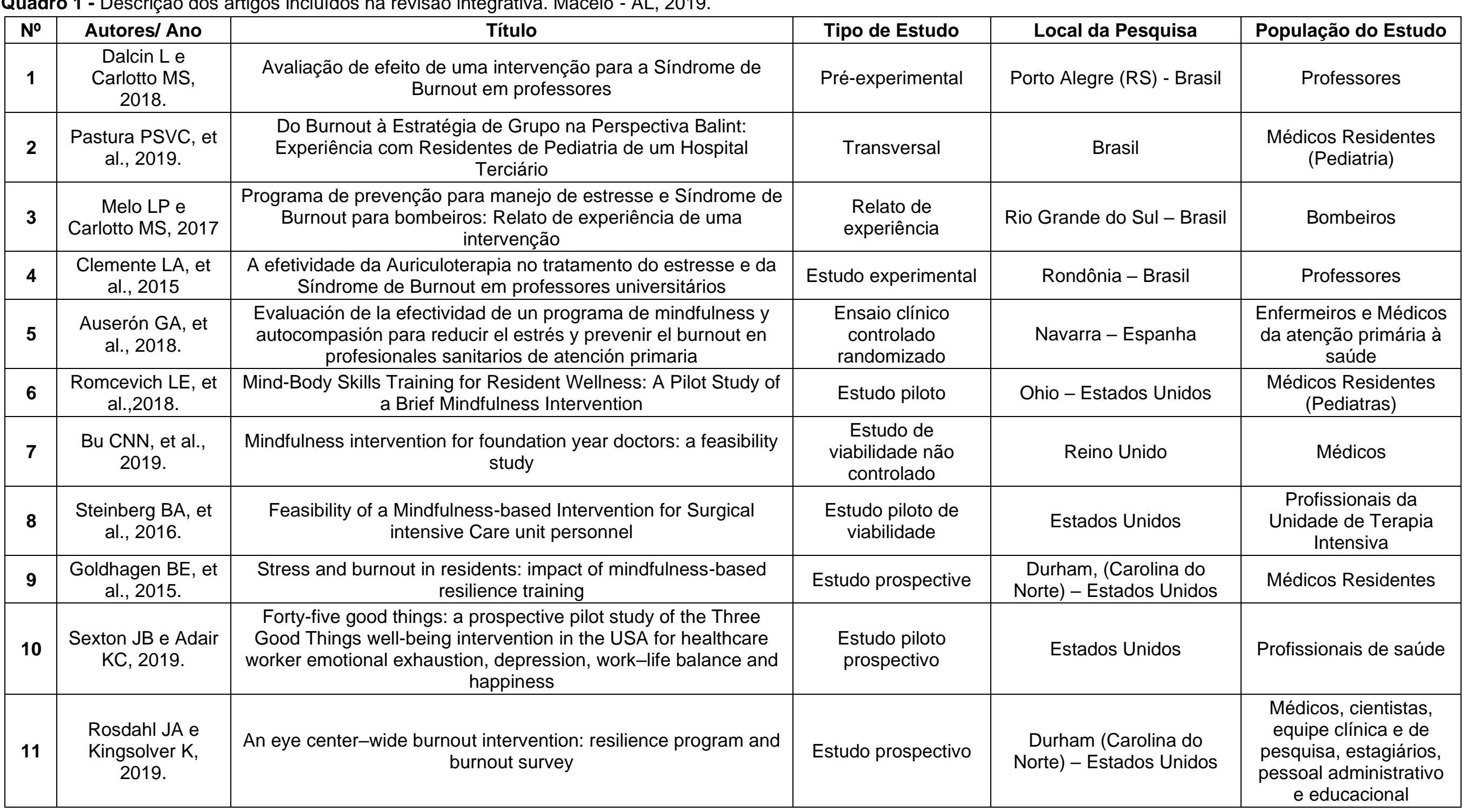


Revista Eletrônica Acervo Saúde / Electronic Journal Collection Health ｜ ISSN 2178-2091

\begin{tabular}{|c|c|c|c|c|c|}
\hline № & Autores/ Ano & Título & Tipo de Estudo & Local da Pesquisa & População do Estudo \\
\hline 12 & $\begin{array}{l}\text { Eriksson T, et al., } \\
2018 .\end{array}$ & $\begin{array}{c}\text { Mindful Self-Compassion Training Reduces Stress and Burnout } \\
\text { Symptoms Among Practicing Psychologists: A Randomized } \\
\text { Controlled Trial of a Brief Web-Based Intervention }\end{array}$ & $\begin{array}{l}\text { Estudo randomizado } \\
\text { controlado }\end{array}$ & Suécia & Psicológos \\
\hline 13 & $\begin{array}{l}\text { Ripp JA, et al., } \\
2016 .\end{array}$ & $\begin{array}{l}\text { A Randomized Controlled Trial to Decrease Job Burnout in First- } \\
\text { Year Internal Medicine Residents Using a Facilitated Discussion } \\
\text { Group Intervention }\end{array}$ & $\begin{array}{l}\text { Estudo randomizado } \\
\text { controlado }\end{array}$ & $\begin{array}{l}\text { Nova lorque - Estados } \\
\text { Unidos }\end{array}$ & Médicos residentes \\
\hline 14 & $\begin{array}{l}\text { Mehrabi T, et al., } \\
\qquad 2015 .\end{array}$ & $\begin{array}{l}\text { The effect of Rosmarinus herbal tea on occupational burnout in } \\
\text { Iran Chemical Industry Investment company employees }\end{array}$ & Estudo experimental & Irã & $\begin{array}{l}\text { Funcionários que } \\
\text { trabalham na seção } \\
\text { técnica }\end{array}$ \\
\hline 15 & $\begin{array}{l}\text { Darban F, et al., } \\
2016 .\end{array}$ & $\begin{array}{l}\text { Effect of Communication Skills Training on the Burnout of } \\
\text { Nurses: A Cross-Sectional Study }\end{array}$ & Transversal & $\begin{array}{l}\text { Iranshahr, Sistan e } \\
\text { Baluchestan - Irã. }\end{array}$ & Enfermeiros \\
\hline 16 & $\begin{array}{l}\text { Ugwoke SC, et } \\
\text { al., } 2018 .\end{array}$ & $\begin{array}{l}\text { A rational-emotive stress management intervention for reducing } \\
\text { job burnout and dysfunctional distress among special education } \\
\text { teachers }\end{array}$ & Estudo de efeito & Nigéria & Professores \\
\hline 17 & $\begin{array}{l}\text { Christopher MS, } \\
\text { et al., } 2018 .\end{array}$ & $\begin{array}{l}\text { Mindfulness-Based Resilience Training to Reduce Health Risk, } \\
\text { Stress Reactivity, and Aggression among Law Enforcement } \\
\text { Officers: A Feasibility and Preliminary Efficacy Trial }\end{array}$ & $\begin{array}{l}\text { Estudo de } \\
\text { viabilidade e eficácia } \\
\text { preliminar }\end{array}$ & $\begin{array}{l}\text { Noroeste do Pacífico } \\
\text { Estados Unidos }\end{array}$ & Policiais \\
\hline 18 & $\begin{array}{l}\text { Bretland RJ e } \\
\text { Thorsteinsson EB, } \\
2015 \text {. }\end{array}$ & $\begin{array}{l}\text { Reducing workplace burnout: the relative benefits of } \\
\text { cardiovascular and resistance exercise }\end{array}$ & $\begin{array}{l}\text { Estudo randomizado } \\
\text { controlado }\end{array}$ & Austrália & Trabalhadores \\
\hline 19 & $\begin{array}{l}\text { Hamilton-west K, } \\
\quad \text { et al., } 2018 .\end{array}$ & $\begin{array}{l}\text { Does a modified mindfulness-based cognitive therapy (MBCT) } \\
\text { course have the potential to reduce stress and burnout in NHS } \\
\text { GPs? Feasibility study }\end{array}$ & $\begin{array}{l}\text { Estudo de } \\
\text { viabilidade }\end{array}$ & Inglaterra & $\begin{array}{l}\text { Profissionais do } \\
\text { Serviço Nacional de } \\
\text { Saúde }\end{array}$ \\
\hline 20 & $\begin{array}{l}\text { Hart } D \text {, et al., } \\
2018 .\end{array}$ & $\begin{array}{c}\text { Does Implementation of a Corporate Wellness Initiative Improve } \\
\text { Burnout? }\end{array}$ & $\begin{array}{l}\text { Estudo de } \\
\text { Intervenção }\end{array}$ & Estados Unidos & Médicos Residentes \\
\hline 21 & $\begin{array}{l}\text { Kashani K, et al., } \\
2015 .\end{array}$ & $\begin{array}{l}\text { Stress and burnout among critical care fellows: preliminary } \\
\text { evaluation of an educational intervention }\end{array}$ & Transversal & $\begin{array}{l}\text { Rochester (Minessota) } \\
\text { Estados Unidos }\end{array}$ & Médicos Residentes \\
\hline 22 & $\begin{array}{l}\text { Bruin El, et al., } \\
\qquad 2017 .\end{array}$ & $\begin{array}{l}\text { Mindful2Work: Effects of Combined Physical Exercise, Yoga, } \\
\text { and Mindfulness Meditations for Stress Relieve in Employees. A } \\
\text { Proof of Concept Study }\end{array}$ & $\begin{array}{l}\text { Estudo de prova de } \\
\text { conceito }\end{array}$ & Holanda & Médicos Residentes \\
\hline
\end{tabular}

Fonte: Silva IS, et al., 2020. 


\section{Revista Eletrônica Acervo Saúde / Electronic Journal Collection Health ISSN 2178-2091}

Quadro 2 - Descrição dos artigos incluídos na revisão integrativa quanto ao instrumento de aferição da Síndrome de Burnout, ao Programa de Intervenção adotado e ao direcionamento da intervenção. Maceió - AL, 2019.

\begin{tabular}{|c|c|c|c|}
\hline № & Instrumento de aferição da SB & Programa de Intervenção / Técnicas Utilizadas & Direcionamento da Intervenção \\
\hline 1 & $\begin{array}{l}\text { Questionário para a Avaliação da Síndrome de } \\
\text { Quemarse por el Trabajo - CESQT-PE }\end{array}$ & Oficinais e envio de material por e-mail & Foco no trabalhador \\
\hline 2 & Maslach Burnout Inventory (MBI) & $\begin{array}{l}\text { Intervenção de grupo na perspectiva Balint, denominado "Grupo de } \\
\text { Escuta". }\end{array}$ & Foco no trabalhador \\
\hline 3 & Não informado & $\begin{array}{l}\text { Oficinais (Exposição teórica; Atividades práticas; Discussão livre e Tarefas } \\
\text { de casa). }\end{array}$ & Foco no trabalhador \\
\hline 4 & Maslach Burnout Inventory (MBI) & Aurículocibernética & Foco no trabalhador \\
\hline 5 & Maslach Burnout Inventory (MBI) & Intervenção baseada na atenção plena e na autopiedade. & Foco no trabalhador \\
\hline 6 & Maslach Burnout Inventory (MBI) & Mind-Body Skills Training (MBST). & Foco no trabalhador \\
\hline 7 & Não informado & $\begin{array}{l}\text { Curso de atenção plena. (adaptação do 'Mindfulness no curso "Local de } \\
\text { trabalho") }\end{array}$ & Foco no trabalhador \\
\hline 8 & Maslach Burnout Inventory & Atenção plena. & Foco no trabalhador \\
\hline 9 & Maslach Burnout Inventory & Técnicas Mindfulness / atenção plena & Foco no trabalhador \\
\hline 10 & Maslach Burnout Inventory & Versão adaptada do 3GT (Three Good Things). & Foco no trabalhador \\
\hline 11 & Maslach Burnout Inventory & $\begin{array}{l}\text { Programa de resiliência desenvolvido pelos autores, que consistia em: (1) } \\
\text { e-mails de bem-estar, (2) apresentações de rodadas gerais, (3) bem-estar } \\
\text { apresentações para professores e (4) "Doctors Lounge" série de discussão. }\end{array}$ & Foco no trabalhador \\
\hline 12 & $\begin{array}{l}\text { Shirom-Melamed Burnout Questionnaire } \\
\qquad(\mathrm{SMBQ})\end{array}$ & $\begin{array}{l}\text { Utilizou-se o Programa de treinamento "Atenção plena e compaixão } \\
\text { consigo mesmo e outros" desenvolvido por Schenström (2017). }\end{array}$ & Foco no trabalhador \\
\hline
\end{tabular}




\section{Revista Eletrônica Acervo Saúde / Electronic Journal Collection Health ｜ ISSN 2178-2091}

\begin{tabular}{|c|c|c|c|}
\hline № & Instrumento de aferição da SB & Programa de Intervenção / Técnicas Utilizadas & Direcionamento da Intervenção \\
\hline 13 & $\begin{array}{l}\text { Maslach Burnout } \\
\text { Inventory }\end{array}$ & Grupos de Discussão sobre estresse, equilíbrio e satisfação no trabalho. & Foco no trabalhador \\
\hline 14 & Geldard occupational burnout questionnaire & Terapia Herbal & Foco no trabalhador \\
\hline 15 & Jackson and Maslach Burnout Inventory & $\begin{array}{l}\text { Oficinas (Palestras, perguntas e respostas, discussão em grupo, exibição } \\
\text { de filmes e tarefas práticas - incluindo a implementação prática de } \\
\text { conteúdos educacionais apresentados na oficina e o fornecimento de } \\
\text { feedback aos participantes). }\end{array}$ & Foco no trabalhador \\
\hline 16 & Shirom-Melamed Burnout Questionnaire & $\begin{array}{c}\text { Manual do Programa Rational Emotive Behavior Therapy (REBT). Técnicas } \\
\text { como como ensino direto, definição de objetivos, relaxamento, } \\
\text { dramatização, disputa, uso de auto-fala racional, técnicas motivacionais de } \\
\text { entrevistas, Questionamento socrático, ensaio cognitivo, técnicas de } \\
\text { dessensibilização, tarefas de casa e técnicas de imagens foram usadas } \\
\text { para ajudar os participantes do estudo. }\end{array}$ & Foco no trabalhador \\
\hline 17 & Oldenburg Burnout Inventory & Mindfulness-Based Resilience Training. & Foco no trabalhador \\
\hline 18 & Maslach Burnout Inventory & $\begin{array}{c}\text { Introdução de exercícios (cardiovasculares e de resistência) a uma } \\
\text { população sedentária. }\end{array}$ & Foco no trabalhador \\
\hline 19 & $\begin{array}{l}\text { Maslach Burnout } \\
\text { Inventory (MBI) }\end{array}$ & Versão modificado do Mindfulness-Based Cognitive Therapy (MBCT). & Foco no trabalhador \\
\hline 20 & Maslach Burnout Inventory & "The Happiness Practice" (THP). & Foco no trabalhador \\
\hline 21 & Maslach Burnout Inventory & $\begin{array}{c}\text { Intervenção adaptada do programa Stress Management and Resiliency } \\
\text { Training (SMART). }\end{array}$ & \\
\hline 22 & Checklist Individual Strength (CIS) & Mindful2Work. & \\
\hline
\end{tabular}

Fonte: Silva IS, et al., 2020.

REAS/EJCH | Vol.12(10) | e4402 | DOI: https://doi.org/10.25248/reas.e4402.2020～Página 8 de 12




\section{Instrumentos de aferição da SB}

Tratando-se da identificação da Síndrome de Burnout, $63,6 \%$ dos estudos analisados utilizaram o Maslach Burnout Inventory (MBI), o referido instrumento é o mais utilizado para diagnosticar a síndrome de burnout em diversas categorias de trabalhadores, sendo considerado padrão ouro na pesquisa clínica e/ou quantificação do esgotamento (MASLACH C, et al., 1996).

O Maslach Burnout Inventory é um questionário autoaplicável, com 22 declarações sobre sentimentos ou atitudes pessoais em relação ao trabalho (ex.: Sinto-me cansado(a) ao final da jornada de trabalho), que abrangem três os 3 aspectos fundamentais da SB: exaustão emocional (EE - 9 questões - 1, 2, 3, 6, 8, 13, 14,16 , e 20 - traduzem sentimentos de se estar emocionalmente exausto e esgotado com o trabalho), realização profissional (RP - oito questões - 4, 7, 9, 12, 17, 18, 19 e 21 - descrevem sentimentos ao nível da capacidade e sucessos alcançados no trabalho com pessoas) e despersonalização (DP - 5 questões - 5, 10, 11, 15 e 22 - descrevem respostas impessoais, consiste na queda de empatia em relação ao paciente).

As respostas são em escala de pontuação do tipo Likert com sete graus de intensidade que vão desde: 1 (nunca), 2 (algumas vezes por ano), 3 (uma vez por mês), 4 (algumas vezes por mês), 5 (uma vez por semana), 6 (algumas vezes por semanas) e 7 (todos os dias). Altos escores em exaustão emocional e despersonalização (subescalas diretas) e baixos escores em realização profissional (subescala inversa) indicam alto nível de burnout (MASLACH C, et al., 1996).

\section{Programas de Intervenção utilizados na prevenção/terapêutica da SB}

Praticamente em todos os estudos analisados, foram empregadas estratégias de prevenção/enfretamento da SB com foco no trabalhador. Os programas com foco no trabalhador têm sido os mais frequentemente adotados (PASTURA PSVC, et al., 2019). Dentre os programas e/ou técnicas de intervenção empregados, o "Mindfulness" ou "atenção plena" foi aplicado em $41 \%(n=9)$ dos estudos analisados. As Oficinas aparecem em segundo lugar, com $13,6 \%(n=3)$ seguidas dos grupos de discussão e das técnicas de manejamento de estresse, ambas com $9,1 \%(n=2)$.

O conceito de mindfulness ou de "atenção plena" fundamenta-se nas tradições contemplativas do Oriente, estando comumente associado às práticas meditativas budistas ${ }^{38} \mathrm{e}$ visam o desenvolvimento da sabedoria $\mathrm{e}$ introspecção (autoavaliação) que auxiliarão no alívio do sofrimento (SIEGAL RD, et al., 2008). Diversos estudos demonstram os benefícios da prática em diversas áreas, seja na prática clínica, na assistência médica e/ou psicológica, no campo educacional, nas relações familiares ou no ambiente organizacional (SEGURA MSG, 2016).

No campo laboral, dentre os programas de intervenção com sustentação empírica relevante, as técnicas de meditação mindfulness têm sido apontadas como uma estratégia de enfrentamento eficaz para o estresse laboral e potencial prevenção/tratamento para Síndrome de Burnout (LEITER MP, et al., 2014).

Também foram empregados como técnicas de intervenção, Oficinas. Os encontros consistiam, basicamente, em: exposição teórica; atividades práticas; discussão livre e tarefas de casa. Esse tipo de intervenção permite que o trabalhador desenvolva habilidades essenciais e indispensáveis como a escuta, 0 enfrentamento de situações de estresse e o aperfeiçoamento do diálogo, além de permitir uma reflexão sobre a finalidade de seu trabalho, sobre as próprias percepções em relação à prática, levando a construção do conhecimento através da troca de experiências (NASCIMENTO LG, et al., 2014).

Ressalta-se que uma das alternativas mais simples para promover a saúde mental no ambiente de trabalho é a utilização de recursos educativos. Estes recursos quando são voltados para ações de promoção da saúde, prevenção de doenças e na vida cotidiana da população, facilitam a incorporação de práticas corretas de forma a atender as reais necessidades (PELICIONI MCF e PELICIONI AF, 2007). Um bom exemplo, são os manuais de orientação destinados à promoção e educação em saúde que trazem importantes contribuições, tais como, apoio pedagógico para a educação continuada dos profissionais de saúde. Além de promover a capacitação e colaborar na uniformização das orientações e estimulam os profissionais para a ação (ECHER IC, 2005). 
É importante salientar que as estratégias de intervenção com foco no indivíduo são relevantes, visto que, ao considerar sua individualidade, ampliam e melhoram as aptidões do trabalhador. No entanto, estudos advertem quanto aos efeitos de intervenções centradas unicamente no trabalhador, pois podem reforçar suas frustrações e baixa autoestima e favorecer o isolamento, mascarando o problema em razão do consenso de que Burnout fundamente-se no contexto do trabalho.

A eficácia da intervenção no combate à Síndrome de Burnout é estabelecida com maior efetividade quando são empregadas estratégias com abordagem individual em conjunto com estratégias organizacionais (MORENO FN, et al., 2011).

Desta forma, para uma abordagem efetiva, os programas de intervenção para prevenção da SB devem ser idealizados por meio de uma temática contextualizada, pois com isso, cria-se uma relação direta entre as necessidades dos sujeitos envolvidos e as instituições laborais, sendo portanto, resultados de uma ação conjunta entre os indivíduos e as organizações, estas por sua vez, devem estar atentas aos sinais que precedem o desenvolvimento desta síndrome (MELO LP e CARLOTTO MS, 2017).

\section{Benefícios dos Programas de Intervenção na SB}

De maneira geral, os programas de intervenção adotados, haja vista que as pessoas que deles participam, frequentemente, apresentam uma redução significativa no desgaste físico e/ou emocional, além de mudanças positivas relacionadas aos fatores de risco da Síndrome de Burnout (AWA WL, et al., 2010).

$\mathrm{Na}$ presente pesquisa, $81 \%$ dos estudos analisados demonstraram algum benefício na prevenção/terapêutica da SB. Resultado semelhante foi encontrado em uma revisão sistemática da literatura, realizada por Awa WL, et al. (2010), que constatou que cerca de $80 \%$ dos 25 programas de intervenção analisados foram úteis na redução do burnout ou de qualquer um de seus principais componentes.

Mesmo diante do crescimento de estudos voltados a esta temática ao longo dos anos, ainda existe poucas produções cientificas referentes a análise de programas de intervenção focados na prevenção da SB. É importante ressaltar que a referida síndrome reflete na desarmonia entre os interesses das instituições laborais e dos profissionais, pois a mesma está intimamente relacionada a satisfação pessoal e ocupacional dos trabalhadores (MELO LP e CARLOTTO MS, 2017).

Os estudos que utilizaram programas de intervenção baseados nas técnicas de mindfulness (ou "atenção plena") mostraram que houve melhora significativa na atitude positiva, no estresse percebido e na resiliência após a intervenção, assim como demonstrou melhoria significativa no esgotamento (despersonalização) e atenção plena (BU CNN, et al., 2019).

$\mathrm{Na}$ área médica, podem contribuir para redução do estresse, da "autofrieza" e do cansaço emocional, melhorando a capacidade de atenção e autopiedade, promovendo maior bem-estar, melhoria da vida profissional e, consequentemente, relacionamentos mais satisfatórios com os pacientes (AUSERÓN GA, et al., 2018; ERIKSSON T, et al., 2018; BU CNN, et al., 2019).

As intervenções realizadas por meio de oficinais, de modo geral, também mostraram impacto em importantes dimensões que previnem a SB nos grupos investigados. Através das técnicas utilizadas, os trabalhadores puderam desenvolver novas habilidades conectadas com a sua atividade laboral (MELO LP e CARLOTTO MS, 2017).

Além disso, puderam experimentar novas maneira de enfrentar os agentes estressores no ambiente de trabalhando, passaram a buscar soluções norteadas no problema e começaram a utilizar maior diversidade de emoções positivas no trabalho, aumentando seu desejo de atingir as metas individuais e de avaliar seu trabalho como fonte de realização (DALCIN L e CARLOTTO MS, 2016).

Quanto os demais programas/técnicas utilizados, os grupos de discussão propiciaram o resgate do profissionalismo e da humanidade, demonstrando um grande benefício no tocante à assistência médica (PASTURA PSVC, et al., 2019). A aurículocibernética e a terapia herbal demonstrou ser uma intervenção efetivamente significativa para o tratamento dos sinais e sintomas relacionados ao estresse e à $S B$ (CLEMENTE LA, et al., 2015; MEHRABI T, et al., 2015). 
A prática de exercícios cardiovasculares e de treinamento resistido foi eficaz no aumento da sensação de bem-estar e de realização pessoal, assim como na diminuição do sofrimento psicológico, do estresse percebido e das emoções e exaustão (BRETLAND RJ e THORSTEINSSON EB, 2015). Por fim, o Three Good THing (3GT) pareceu promissor, de baixo custo e breve intervenção para melhorar o bem-estar dos profissionais de saúde, pois os participantes do estudo exibiram significantes melhorias da linha de base na exaustão emocional, sintomas de depressão e felicidade e no equilíbrio entre vida profissional e pessoal (SEXTON JB e ADAIR KC, 2019).

\section{CONSIDERAÇÕES FINAIS}

Estudos sobre a SB têm crescido ao longo dos anos, o que representa uma área promissora e de crescimento rápido, sobretudo na comunidade cientifica internacional. Apesar de ser um tema ainda pouco explorado no Brasil, vale destacar que houve uma equivalência no tocante aos instrumentos utilizados para detecção e prevenção da SB nos estudos que compuseram a presente pesquisa. À guisa de síntese, ressaltase a necessidade de novos estudos, principalmente com intervenções com foco na organização e/ou mistas, assim como estudos de abordagem qualitativa e quantitativa, pois existe uma carência dessas pesquisas com foco nestes aspectos, tanto no âmbito nacional quanto internacional.

\section{REFERÊNCIAS}

1. AUSERÓN GA, et al. Evaluación de la efectividad de un programa de mindfulness y autocompasión para reducir el estrés y prevenir el burnout en profesionales sanitarios de atención primaria. Aten Primaria, 2018; 50(3):141-150.

2. AWA WL, et al. Burnout prevention: A review of intervention programs. Patient Education and Counseling, 2010; 78(2):184-190.

3. BRASIL. Decreto no 3.048, de 6 de maio de 1999. In: Aprova o Regulamento da Previdência Social, e dá outras providências.

4. BRETLAND RJ, THORSTEINSSON EB.Reducing workplace burnout: the relative benefits of cardiovascular and resistance exercise. PeerJ, 2015; 3: e891.

5. BRUIN El, et al. Mindful2Work: Effects of Combined Physical Exercise, Yoga, and Mindfulness Meditations for Stress Relieve in Employees. A Proof of Concept Study. Mindfulness (N Y), 2017; 8:204-217.

6. BU CNN, et al. Mindfulness intervention for foundation year doctors: a feasibility study. Pilot Feasibility Stud. Stud de Viabilidade Piloto, 2019; 5: 61

7. BUEHLER AM, et al. Como avaliar criticamente um ensaio clínico de alocação aleatória em terapia intensiva. Rev Bras Ter Intensiva, 2009; 21(2):219-225.

8. CHRISTOPHER MS, et al. Mindfulness-Based Resilience Training to Reduce Health Risk, Stress Reactivity, and Aggression among Law Enforcement Officers: A Feasibility and Preliminary Efficacy Trial. Psychiatry Res., 2018; 264:104-115.

9. CLEMENTE LA, et al. A efetividade da Auriculoterapia no tratamento do estresse e da Síndrome de Burnout em professores universitários. Cad. Naturol. Terap. Complem., 2015; 4(7):21-27.

10. DALCIN L, CARLOTTO MS. Avaliação de efeito de uma intervenção para a Síndrome de Burnout em professores. Psicol. Esc. Educ., 2018; 22(1):141-150.

11. ECHER IC. Elaboração de manuais de orientação para o cuidado em saúde. Rev. latinoam. enferm., 2005; 13(5):754757.

12. ERIKSSON T, ET al.Mindful Self-Compassion Training Reduces Stress and Burnout Symptoms Among Practicing Psychologists: A Randomized Controlled Trial of a Brief Web-Based Intervention.Front Psychol., 2018; 9: 2340.

13. GOLDHAGEN BE, et al. Stress and burnout in residents: impact of mindfulness-based resilience training. Adv Med Educ Pract.,2015;6:525-532.

14. HART D, et al. Does Implementation of a Corporate Wellness Initiative Improve Burnout? West J Emerg Med., 2019; 20(1):138-144.

15. HERNANDEZ EG, et al. Beneficios de Mindfulness sobre el estrés laboral. 2017. In: Congreso Prevencionar 2017.

16. INTERNATIONAL LABOUR ORGANIZATION. 2016. In: Workplace stress. A collective challenge. World day for safety and health at work 28 april 2016.

17. ISMA BR- International Stress Management Association. 2017. In: Leia antes de fritar.

18. KASHANI K, et al. Stress and burnout among critical care fellows: preliminary evaluation of an educational intervention. Med Educ Online., 2015; 20: 27840.

19. LEITER MP, et al. Burnout at work: a psychological perspective. Nova lorque, NY: Psychology Press, 2014; 174p.

20. LIMA AFG, SENE AR. Mindfulness nas terapias de redução da ansiedade. Rev. Psicol Saúde e Debate, 2017; 3(Supl. 1):40-41.

21. MASLACH C, et al. Maslach Burnout Inventory. 3rd ed. Palo Alto: Consulting Psychologists Press, 1996.

22. MASLACH C, et al. Measuring burnout. 2008. In: Cooper CL, Cartwright S (eds). The Oxford handbook of organizational well-being. 
23. MEHRABI T, et al. The effect of Rosmarinus herbal tea on occupational burnout in Iran Chemical Industry Investment company employees. Iran J Nurs Midwifery Res., 2015; 20(4):460-464.

24. MELO LP, CARLOTTO MS. Programa de prevenção para manejo de estresse e Síndrome de Burnout para bombeiros: Relato de experiência de uma intervenção. Estud. psicol., 2017; 22(1):99-108.

25. MORENO FN, et al. Estratégias e intervenções no enfrentamento da Síndrome de Burnout. Rev. enferm., 2011; 19(1):140-5.

26. NASCIMENTO LG, et al. Oficinas de intervenção psicossocial com agentes comunitários de saúde: reflexões e intervenções dialogadas. Rev Bras Med Fam Comunidade, 2014; 9(33):336-342.

27. OMS- Organização Mundial da Saúde. 2019a. In: CID-11para Estatísticas de Mortalidade e Morbidade(Versão: 04/2019). QD85 Burn-out.

28. OMS-Organização Mundial da Saúde. 2019b. In: Saúde mental no local de trabalho. Folha de Informação.

29. OPAS- Organização Pan-Americana de Saúde. 2016. In: Determinantes sociais e riscos para a saúde, doenças Crônicas não transmissíveis e saúde mental. Estresse no ambiente de trabalho cobra preço alto de indivíduos, empregadores e sociedade.

30. PASTURA PSVC, et al. Do Burnout à Estratégia de Grupo na Perspectiva Balint: Experiência com Residentes de Pediatria de um Hospital Terciário. Rev. bras. educ. med., 2019; 43(2):32-39.

31. PELICIONI MCF, PELICIONI AF. Educação e promoção da saúde: uma retrospectiva histórica. Mundo saúde (Impr.), 2007;31(3):320-328.

32. PEREIRA AMTB. Burnout: Quando o trabalho ameaça o bem estar do trabalhador. $3^{a}$ ed. São Paulo: Casa do Psicólogo, 2010; 282p.

33. RAHAL GM. Atenção plena no contexto escolar: benefícios e possibilidades de inserção. Psicol. esc. educ., 2018; 22(2):347-358.

34. RIPP JA, et al. A Randomized Controlled Trial to Decrease Job Burnout in First-Year Internal Medicine Residents Using a Facilitated Discussion Group Intervention. J Grad Med Educ., 2016; 8(2):256-259.

35. ROSDAHL JA, KINGSOLVER K. An eye center-wide burnout intervention: resilience program and burnout survey. Digit J Ophthalmol, 2019; 25(1):5-11.

36. RUBACK SP, et al. Estresse e Síndrome de Burnout em Profissionais de Enfermagem que Atuam na Nefrologia: Uma Revisão Integrativa. J. res.: fundam. care., 2018; 10(3): 889-899.

37. SEGURA MGS. Beneficios del mindfulness y su influencia en las relaciones familiares: Un estudio de caso. Alternativas en psicologia, 2016; 34:121-131.

38. SEXTON JB, ADAIR KC. Forty-five good things: a prospective pilot study of the Three Good Things well-being intervention in the USA for healthcare worker emotional exhaustion, depression, work-life balance and happiness. BMJ Open, 2019; 9(3): e022695.

39. SIEGAL RD, et al. Mindfulness: What is it? Where does it come from? 2008. In: Didonna F. (Ed.). Clinical Handbook of Mindfulness.

40. UGWOKE SC, et al. A rational-emotive stress management intervention for reducing job burnout and dysfunctional distress among special education teachers. Medicine, 2018; 97(17):e0475. 